\title{
Genetic variants in FBLIM1 gene do not contribute to SAPHO syndrome and chronic recurrent multifocal osteomyelitis in typical patient groups
}

Gunter Assmann ${ }^{1}$, Michaela Köhm², Volker Schuster ${ }^{3}$, Frank Behrens², Rotraut Mössner ${ }^{4}$, Nina Magnolo ${ }^{5}$, Vinzenz Oji ${ }^{5}$, Harald Burkhardt ${ }^{2}$ and Ulrike Hüffmeier ${ }^{6^{*}}$ (D)

\begin{abstract}
Background: Syndrome of synovitis acne pustulosis hyperostosis osteitis (SAPHO) and chronic recurrent multifocal osteomyelitis (CRMO) present two diseases of a dermatologic and rheumatologic spectrum that are variable in manifestation und therapeutic response. Genetic risk factors have long been assumed in both diseases, but no single reliable factor has been identified yet. Therefore, we aimed to clinically characterize a patient group with syndrome of synovitis acne pustulosis hyperostosis osteitis $(\mathrm{SAPHO})(n=47)$ and chronic recurrent multifocal osteomyelitis (CRMO)/ chronic non-bacterial osteomyelitis (CNO) $(n=9)$ and analyze a CRMO candidate gene.
\end{abstract}

Methods: Clinical data of all patients were collected and assessed for different combinations of clinical symptoms. $\mathrm{SAPHO}$ patients were grouped into categories according to the acronym; disease-contribution by pathogens was evaluated. We sequenced coding exons of FBLIM1.

Results: Palmoplantar pustular psoriasis (PPP) was the most common skin manifestation in CRMO/CNO and SAPHO patients; most SAPHO patients had sterno-costo-clavicular hyperostosis. The most common clinical category of the acronym was S_PHO $(n=26)$. Lack of pathogen detection from bone biopsies was more common than microbial isolation. We did not identify autosomal-recessive FBLIM1 variants.

Conclusions: S_PHO is the most common combination of symptoms of its acronym. Genetic analyses of FBLIM1 did not provide evidence that this gene is relevant in our patient group. Our study indicates the need to elucidate SAPHO's and CRMO/CNO's pathogenesis.

Keywords: Chronic recurrent multifocal osteomyelitis (CRMO), Chronic non-bacterial osteomyelitis (CNO), Syndrome of synovitis acne pustulosis hyperostosis osteitis (SAPHO (syndrome)), Coding variants, Association

\footnotetext{
* Correspondence: ulrike.hueffmeier@uk-erlangen.de

${ }^{6}$ Institute of Human Genetics, University Hospital Erlangen,

Friedrich-Alexander-Universität Erlangen-Nürnberg, Schwabachanlage 10,

91054 Erlangen, Germany

Full list of author information is available at the end of the article
}

(c) The Author(s). 2020 Open Access This article is licensed under a Creative Commons Attribution 4.0 International License, which permits use, sharing, adaptation, distribution and reproduction in any medium or format, as long as you give appropriate credit to the original author(s) and the source, provide a link to the Creative Commons licence, and indicate if changes were made. The images or other third party material in this article are included in the article's Creative Commons licence, unless indicated otherwise in a credit line to the material. If material is not included in the article's Creative Commons licence and your intended use is not permitted by statutory regulation or exceeds the permitted use, you will need to obtain permission directly from the copyright holder. To view a copy of this licence, visit http://creativecommons.org/licenses/by/4.0/ The Creative Commons Public Domain Dedication waiver (http://creativecommons.org/publicdomain/zero/1.0/) applies to the data made available in this article, unless otherwise stated in a credit line to the data. 


\section{Background}

Syndrome of synovitis acne pustulosis hyperostosis osteitis (SAPHO) belongs to a group of rare, variable, chronic autoinflammatory diseases of the skeleton often in combination with certain skin manifestations and typically manifests in early adulthood [1-3]. SAPHO syndrome's manifestations of the skeleton are manifold; however, osteitis with hyperostosis is considered as a pathognomonic lesion of SAPHO syndrome [3]. Moreover, it can present as a sclerosing osteitis originating from the bone marrow, a hyperostosis with increased bone cuff formed by the periost, an ossification of ligaments, accompanied by osteolysis or an erosion of a joint. Predilection sites of the disease are joints/ bones of the anterior chest wall, mostly sterno-costo-clavicular structures. As skeletal manifestations in SAPHO syndrome often affect the spine and have common features with ankylosing spondylitis and psoriatic arthritis (PsA), it has been considered to belong to the spondylarthropathies [3].

Chronic recurrent multifocal osteomyelitis (CRMO) has a similar clinical spectrum, and the typical range of manifestation in CRMO is childhood [4-6]. CRMO lesions affect most frequently the lower extremity, the vertebrae, pelvic girdle and the foot [6]. Chronic nonbacterial osteomyelitis $(\mathrm{CNO})$ is the more comprehensive name for this entity, so rarely used, while comprising milder/ unifocal osteomyelitis forms also [7].

Palmoplantar pustular psoriasis (PPP) is the most common skin manifestation in CRMO and SAPHO syndrome $[1,8]$. Although the further typical skin manifestation of acne contributed to the name SAPHO syndrome, it is less frequently observed, at least simultaneously, while often prior to skeletal manifestations. SAPHO syndrome patients can also manifest with the more common psoriasis form, psoriasis vulgaris (PsV). Similarly as in CRMO, the frequency of Crohn's disease is increased in SAPHO $[1,8,9]$ and considerably higher than one might expect when combining the prevalences of the two diseases. The significant clinical overlap between CRMO and SAPHO causes recurrent discussions whether CRMO represents the pediatric manifestation of SAPHO; and a transition from CRMO to adult SAPHO syndrome has been described in several cases $[5,10-12]$.

The etiology of CRMO and SAPHO syndrome is unsolved. Current hypotheses in SAPHO suggest a genetic predisposition in combination with a bacterial infection, resulting in reactive osteitis $[3,13]$. Interestingly, the pathogen Propionibacterium acnes could be isolated in patients of several independent studies, e.g. [1, 8, 9, 14]. In previous genetic studies of SAPHO, single candidate genes - partially known to be causal in clinically overlapping, but syndromic forms (e.g. Majeed syndrome) or in mice models of the diseases - were analyzed in single patients/ families with SAPHO or SAPHO similar symptoms, but disease-causing mutations were not identified $[15,16]$.

More recently, bi-allelic rare variants of FBLIM1 encoding filamin binding LIM protein 1 were identified in two CRMO patients: one homozygous missense variant was selected as the most plausible candidate of homozygous variants in 22 genes [17]. One of further 96 CRMO patients was a compound-heterozygous carrier of a heterozygous frameshift variant/ an intronic variant located (rs41310367) in a putative enhancer. An intronic FBLIM1 variant was observed to be more frequent in CRMO patients than in the general population (rs114077715). Findings of this new potential candidate gene FBLIM1 in CRMO prompted us to analyze our cohort of CRMO and SAPHO syndrome patients for rare coding variants in FBLIM1.

\section{Methods}

All patients were of European origin and diagnosed with $\mathrm{CRMO} / \mathrm{CNO}$ or SAPHO syndrome at German university hospitals by board certified rheumatologists, pediatricians, specialized in pediatric rheumatology $(n=51)$, or by dermatologists $(n=5)$. Data on clinical characteristics were collected. For the assignment of SAPHO syndrome patients to the different combinations of its acronym, we omitted two patients due to lack of some essential data. Study approval was obtained through the ethical committees of the Universities of Erlangen, Frankfurt, Homburg and Göttingen; all individuals provided their written informed consent and in the case of minors, written informed consent was obtained from their legal guardians. All investigations were conducted according to the Declaration of Helsinki principles.

Coding exons and adjacent intronic sequences of FBLIM1 were sequenced by Sanger using intron based primers (Supplementary Table 1, Supplementary Fig. 1, Supplementary Table 3) as described recently [18]. Published candidate variants of a frequency of $>2 \%$ prompted us to consider all variants with a minor allele frequency (MAF) of < $3 \%$; we compared their frequency with the frequency of the largest publicly available group of European controls (gnomAD) [19] (https://gnomad. broadinstitute.org/) using allele frequency distribution tests.

\section{Results}

\section{Clinical characteristics of patient groups}

The majority of $9 \mathrm{CRMO} / \mathrm{CNO}$ and 47 SAPHO syndrome patients were female $(n=35 ; 62 \%)$. The average age of onset in CRMO/ CNO patients was $12.2( \pm 4.6)$ years, in SAPHO syndrome $40.2( \pm 14.1)$ years (Supplementary Table $2 \mathrm{~A})$. The majority of all patients had PPP ( $n=38 ; 68 \%$; Fig. 1a), $24 \%$ acne. 
A)

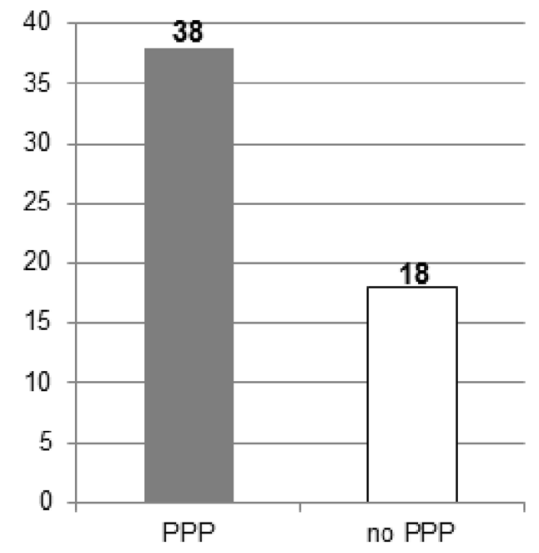

C)

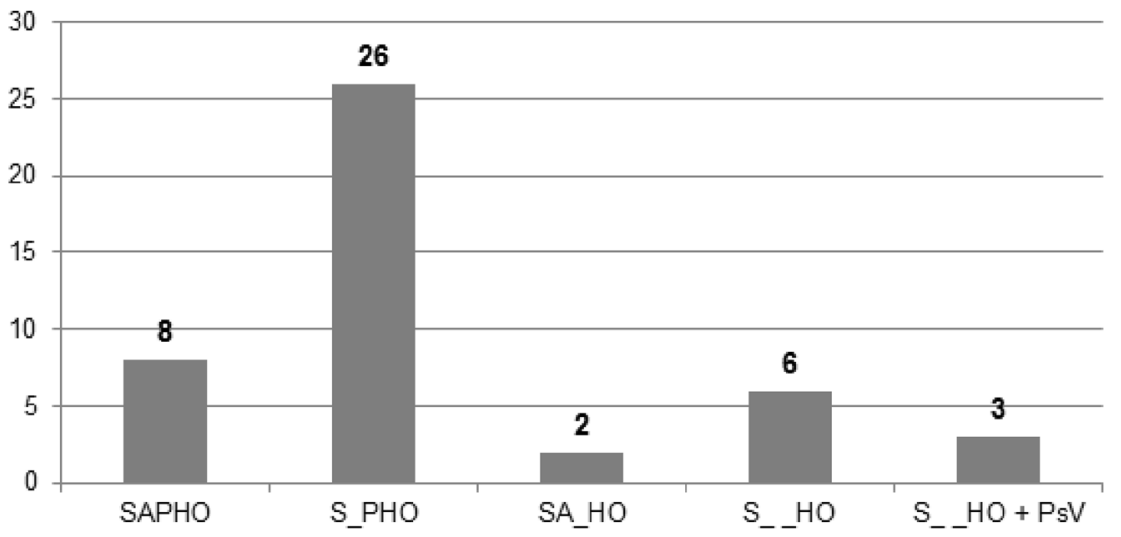

D)

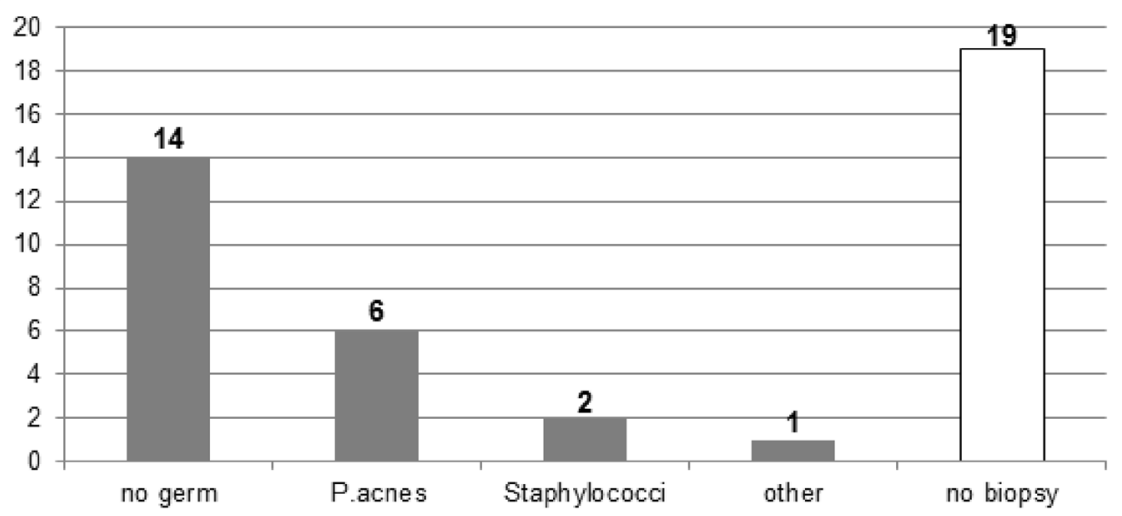

B)

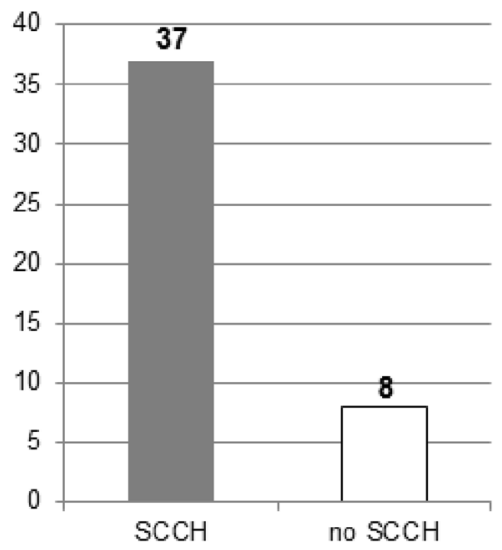

Fig. 1 Clinical characteristics in CRMO and SAPHO syndrome patients. a Number of patients with/ without palmoplantar pustular psoriasis (PPP). b Number of SAPHO syndrome patients with sterno-costo-clavicular hyperostosis (SCCH). c Number of SAPHO syndrome patients fulfilling all or part of the clinical characteristics included in the acronym SAPHO (syndrome of synovitis acne pustulosis hyperostosis osteitis). $\mathbf{d}$ Number of bone biopsies performed in 23 SAPHO syndrome patients with (lack of) detection of germs and number of patients $(n=19)$ who did not have bone biopsies. . . acnes $=$ Proprionibacterium acnes.

Eight CRMO/ CNO patients had multifocal osteomyelitis; and frequencies of skin manifestations are described in Supplementary Table 2B. Two CRMO patients had 


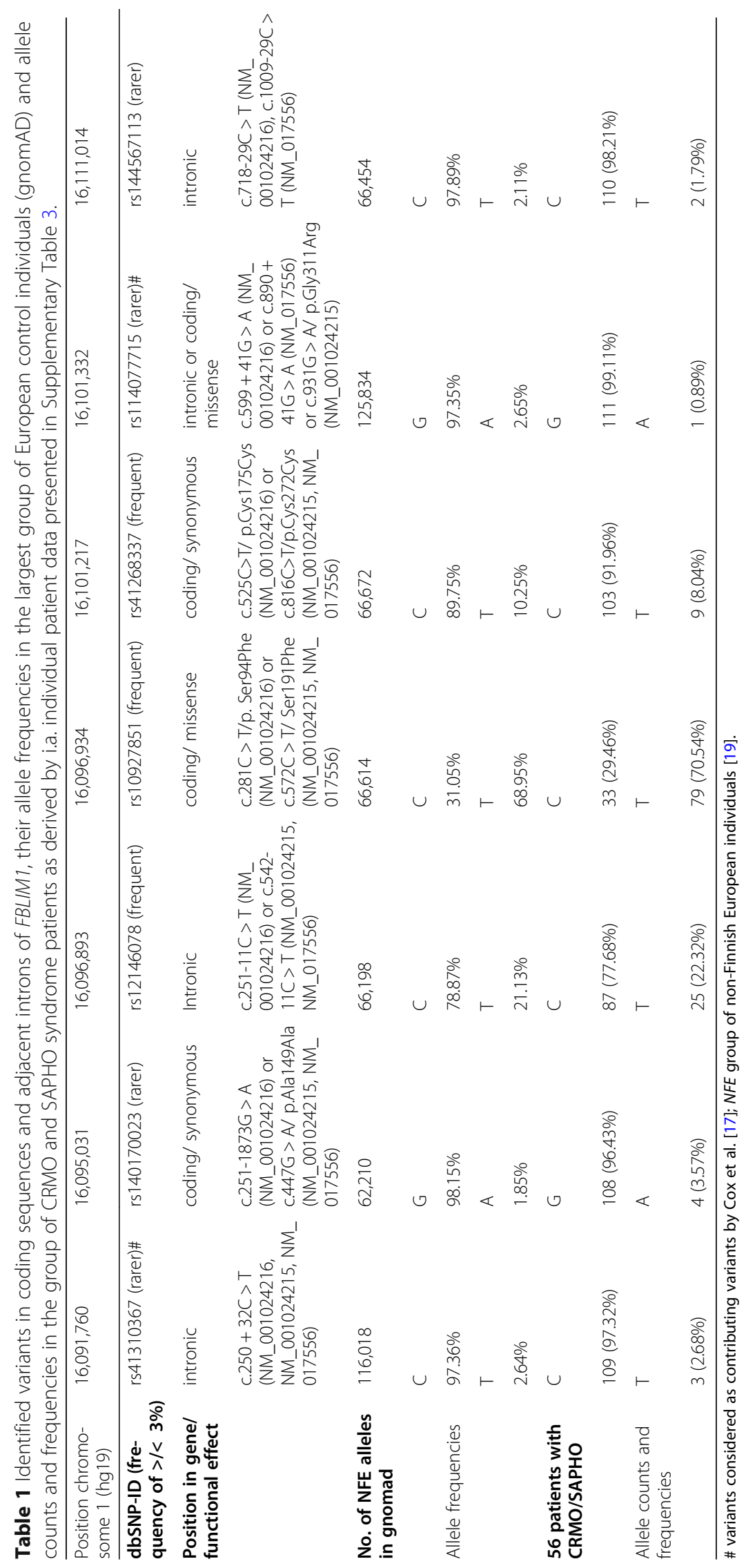


$82 \%$, Fig. 1b; Supplementary Table 2A). When considering typical manifestations of SAPHO designating its acronym (syndrome of synovitis acne pustulosis hyperostosis osteitis), $58 \%$ of 45 patients $(n=26$; Fig. 1c) matched the category of S_PHO, $18 \%(n=8)$ the full acronym and $4 \%(n=2)$ SA_HO. $13 \%$ of patients $(n=6)$ did neither have PPP nor acne, while in $7 \%$ of patients $(n=3)$, plaque type psoriasis as a single manifestation was diagnosed. PsV was more commonly observed in patients also presenting with PPP $(n=8)$ than as a single skin manifestation $(n=3)$. Arthritis was commonly diagnosed in $69 \%$ of SAPHO syndrome patients. In SAPHO syndrome patients, $74 \%$ (25 of 34 ) of patients with PPP had additional arthritis, while arthritis without PPP was observed in $55 \%$ (6 of 11). While data on family history was not available for many patients, most SAPHO syndrome patients had a negative family history. In $26 \%$ of patients with detectable germ in bone biopsy $(n=23), P$. acnes was identified, while germs were not discovered in $61 \%$ of biopsied patients (Fig. 1d).

\section{Analysis of variants in the FBLIM1 gene}

We sequenced FBLIM1 with a genotyping rate of $100 \%$ (Supplementary Table 3). We did not identify any rare $(<0.1 \%)$ or truncating variant. Three variants had a MAF of $>3 \%$ (Table 1 ) with a similar allele frequency in patients compared to control individuals [19]. Four further variants had a MAF of $1.85-2.65 \%$ in the control individuals. Two variants were located in introns, at positions of -32 (rs41310367) and -29 (rs144567113). A further variant was synonymous (c.447G > A/ p.Ala149Ala; rs140170023) at a phylogenetically not conserved position. Last but not least, we identified rs114077715, a variant that is non-synonymous in a single (NM_001024215) of several isoforms of FBLIM1. Carriers of RARE variants were exclusively SAPHO syndrome patients.

All but one carrier of the rarer variants carried a single variant, while one patient carried the two variants rs41310367 and rs140170023 in heterozygous state, respectively. Although we cannot exclude a functional role of the second variant, a synonymous variant is not an obvious functional candidate. The overall frequency of all identified variants was comparable to a large group of European control individuals (62,210-125,834 NonFinnish European alleles; Table 1) [19].

\section{Discussion}

The clinical picture in CRMO and SAPHO syndrome has previously been described as variable, while certain features show comparable frequencies [1, 4-6, 8, 20]. Concordantly, most of our patients had $\geq 1$ additional skin manifestation. The finding of the most common combination "S_PHO" might be related to our predominant recruitment by rheumatologists. Recently, we observed that by recruiting PPP patients by dermatologists, $25 \%$ of patients had additional arthritis [21]. When applying previous established diagnostic criteria for SAPHO [2, 3], these PPP patients could be diagnosed to have SAPHO and correspond to a subgroup of patients with synovitis and PPP $\left(\mathrm{S}_{-} \mathrm{P}_{-}{ }_{-}\right)$. Interestingly, we obtained evidence that several manifestations (PPP, PsV, arthritis) in SAPHO syndrome manifest more often in combination than as single symptoms. Overall, the distribution of clinical symptoms in our study is comparable to previous patient groups and therefore representative for these diseases.

In contrast to the previous study on FBLIM1, we did not identify any rare missense or truncating variants or any evidence for carriers of two decent candidate variants. Previously, rs114077715 or a genetic variant in linkage disequilibrium (LD) was suggested to be a potential disease-contributing variant in carriers [17], while the lower frequency of this variant in patients compared to controls in our analyses does not confirm the variant's or an LD-dependent variant's relevance. Cox et al. [17] provided evidence that the rare allele of the other variant rs41310367 reduces binding to a transcription factor using in vitro experiments. Our data does not exclude the minor allele of rs41310367 as a potential diseasecontributing variant in carriers, but our findings do not implicate a major role of this variant and indicate lack of other candidate variants in coding/ near-coding regions of the gene in this independent patient group. The South-Asian origin of the two patients carrying more critical variants [17] might suggest a relevance of this gene in patients of Asian, rather than of European origin, while frequencies of the rare SNPs identified in this study are comparable in South-Asian probands in gnomAD [19].

Cox et al. [17] considered FBLIM1 as a suitable candidate gene for CRMO due to a murine knockout model [22]. In those mice, loss of the protein Fblim1 impaired growth and survival of bone marrow stromal cells in vitro, increased osteoclast differentiation in vivo and the level of receptor activator of nuclear factor $\kappa B$ ligand (RANKL), suggesting that Fblim1 might be a major regulator of bone homeostasis. Current findings in human patients with SAPHO syndrome and CRMO combining the previous study and our one - indicate that only a minority of patients carries genetic variants in the corresponding human gene that are functional.

We cannot exclude that lack of confirmation of FBLIM1 as a relevant gene might be due to our smaller patient group or smaller proportion of CRMO patients. Still, when considering SAPHO syndrome and CRMO as part of the same disease spectrum, our study does not support FBLIM1 as a disease gene. As the pathogenesis 
of CRMO and SAPHO syndrome is not well understood, further genetic and immunologic studies are needed to elucidate their molecular basis. This will be fundamental for therapeutic strategies.

\section{Conclusions}

Our study indicates that the combination of symptoms representing S_PHO instead of SAPHO is the most common subcategory. Autosomal-recessive variants in the FBLIM1 gene did not play a role in our typical patients.

\section{Supplementary information}

Supplementary information accompanies this paper at https://doi.org/10. 1186/s12881-020-01037-7.

\section{Additional file 1.}

\section{Abbreviations}

CRMO: Chronic recurrent multifocal osteomyelitis; gnomAD: Genome Aggregation Database (gnomad.broadinstitute.org); FBLIM1: Filamin binding LIM protein 1; MAF: Minor allele frequency; P. acnes: Proprionibacterium acnes; PPP: Palmoplantar pustular psoriasis; PsA: Psoriatic arthritis; PsV: Psoriasis vulgaris; SAPHO syndrome: Syndrome of synovitis acne pustulosis hyperostosis osteitis; SCCH: Sterno-costo-clavicular hyperostosis

\section{Acknowledgements}

We are grateful to all patients participating in this study. We thank Anne Gerschütz for excellent technical assistance.

\begin{abstract}
Authors' contributions
All authors contributed to the study conception and design. Material preparation, data collection and/ or analysis were performed by GA, MK, VS, $\mathrm{FB}, \mathrm{RM}, \mathrm{NM}, \mathrm{VO}, \mathrm{HB}$ and $\mathrm{UH}$. The first draft of the manuscript was written by $\mathrm{UH}$, and all authors commented on previous versions of the manuscript. All authors read and approved the final manuscript.
\end{abstract}

\section{Funding}

The study was partly supported by a grant to U.H. from the Bundesministerium für Bildung und Forschung [BMBF Metarthros 01EC1407A] and by a grant to U.H. from the Interdisciplinary Centre for Clinical Research (laboratory rotation) of the Clinical Center Erlangen of the Friedrich-Alexander-Universität Erlangen-Nürnberg. H.B. and F.B. received funding from the BMBF [ArthroMark (project 4, 01EC1401C). The funders supported positions of single scientists and technicians as well as material resources for the project.

\section{Availability of data and materials}

The datasets generated and/or analyzed during the current study are available in this manuscript and its supplementary information files, see also Supplementary Table 3 and Supplementary Fig. 1. We used data of the publicly available database "Genome Aggregation Database (gnomAD)" to assess the frequency of the largest publicly available group of Europeans [19] (https://gnomad.broadinstitute.org/). Datasets used and/ or analyzed during the current study are available from the corresponding author on reasonable request.

\section{Ethics approval and consent to participate}

Study approval was obtained through the ethical committees of the Universities of Erlangen (209_17B and 463_18B), Frankfurt (199/15), Homburg (275/16) and Göttingen (19/7/13); all individuals provided their written informed consent and in the case of minors, written informed consent was obtained from their legal guardians. All investigations were conducted according to the Declaration of Helsinki principles.
Consent for publication

Not applicable.

\section{Competing interests}

All authors declare that there are no competing interests.

\section{Author details}

'Department of Internal Medicine I, José-Carreras Centrum for Immuno- and Gene Therapy, University of Saarland Medical School, /Saar, Homburg, Germany. ${ }^{2}$ Division of Rheumatology and IME, Fraunhofer Institute, Branch for Translational Medicine and Pharmacology, Goethe University, Frankfurt am Main, Germany. ${ }^{3}$ Hospital for Children and Adolescents, University of Leipzig, Leipzig, Germany. ${ }^{4}$ Department of Dermatology, Georg-August-University Göttingen, Göttingen, Germany. ${ }^{5}$ Department of Dermatology, University of Münster, Münster, Germany. Institute of Human Genetics, University Hospital Erlangen, Friedrich-Alexander-Universität Erlangen-Nürnberg, Schwabachanlage 10, 91054 Erlangen, Germany.

Received: 21 November 2019 Accepted: 28 April 2020

Published online: 12 May 2020

\section{References}

1. Chamot AM, Benhamou CL, Kahn MF, Beraneck L, Kaplan G, Prost A. Acnepustulosis-hyperostosis-osteitis syndrome. Results of a national survey. 85 cases. Revue du rhumatisme et des maladies osteo-articulaires. 1987:54(3): 187-96.

2. Benhamou CL, Chamot AM, Kahn MF. Synovitis-acne-pustulosis hyperostosis-osteomyelitis syndrome (SAPHO). A new syndrome among the spondyloarthropathies? Clin Exp Rheumatol. 1988;6(2):109-12.

3. Assmann G, Simon P. The SAPHO syndrome--are microbes involved? Best Pract Res Clin Rheumatol. 2011;25(3):423-34

4. Bjorksten B, Gustavson KH, Eriksson B, Lindholm A, Nordstrom S. Chronic recurrent multifocal osteomyelitis and pustulosis palmoplantaris. J Pediatr. 1978:93(2):227-31.

5. Jurik AG, Helmig O, Ternowitz T, Moller BN. Chronic recurrent multifocal osteomyelitis: a follow-up study. J Pediatr Orthop. 1988;8(1):49-58.

6. Silier CCG, Greschik J, Gesell S, Grote V, Jansson AF. Chronic non-bacterial osteitis from the patient perspective: a health services research through data collected from patient conferences. BMJ Open. 2017;7(12):e017599.

7. Hofmann SR, Kapplusch F, Mabert K, Hedrich CM. The molecular pathophysiology of chronic non-bacterial osteomyelitis (CNO)-a systematic review. Mol Cell Pediatr. 2017;4(1):7.

8. Hayem G, Bouchaud-Chabot A, Benali K, Roux S, Palazzo E, SilbermannHoffman O, et al. SAPHO syndrome: a long-term follow-up study of 120 cases. Semin Arthritis Rheum. 1999;29(3):159-71.

9. Hurtado-Nedelec M, Chollet-Martin S, Nicaise-Roland P, Grootenboer-Mignot $\mathrm{S}$, Ruimy $\mathrm{R}$, Meyer $\mathrm{O}$, et al. Characterization of the immune response in the synovitis, acne, pustulosis, hyperostosis, osteitis (SAPHO) syndrome. Rheumatology (Oxford). 2008:47(8):1160-7.

10. Kahn MF, Hayem F, Hayem G, Grossin M. Is diffuse sclerosing osteomyelitis of the mandible part of the synovitis, acne, pustulosis, hyperostosis, osteitis (SAPHO) syndrome? Analysis of seven cases. Oral Surg Oral Med Oral Pathol. 1994;78(5):594-8.

11. Tlougan BE, Podjasek JO, O'Haver J, Cordova KB, Nguyen XH, Tee R, et al. Chronic recurrent multifocal osteomyelitis (CRMO) and synovitis, acne, pustulosis, hyperostosis, and osteitis (SAPHO) syndrome with associated neutrophilic dermatoses: a report of seven cases and review of the literature. Pediatr Dermatol. 2009:26(5):497-505.

12. Morbach H, Girschick HJ. Chronic non-bacterial osteomyelitis in childhood a comprehensive review. Curr Rheumatol Rev. 2013:9(1):17-21.

13. Schilling F. Why "spondylodiscitis," why "SAPHO syndrome"? J Rheumatol. 2003;30(3):636 author reply 7

14. Edlund E, Johnsson U, Lidgren L, Pettersson H, Sturfelt G, Svensson B, et al. Palmoplantar pustulosis and sternocostoclavicular arthro-osteitis. Ann Rheum Dis. 1988;47(10):809-15.

15. Ferguson PJ, Lokuta MA, El-Shanti HI, Muhle L, Bing X, Huttenlocher A. Neutrophil dysfunction in a family with a SAPHO syndrome-like phenotype. Arthritis Rheum. 2008;58(10):3264-9.

16. Hurtado-Nedelec M, Chollet-Martin S, Chapeton D, Hugot JP, Hayem G, Gerard B. Genetic susceptibility factors in a cohort of 38 patients with 
SAPHO syndrome: a study of PSTPIP2, NOD2, and LPIN2 genes. J Rheumatol. 2010;37(2):401-9.

17. Cox AJ, Darbro BW, Laxer RM, Velez G, Bing X, Finer AL, et al. Recessive coding and regulatory mutations in FBLIM1 underlie the pathogenesis of chronic recurrent multifocal osteomyelitis (CRMO). PLoS One. 2017;12(3): e0169687.

18. Sighart R, Rech J, Hueber A, Blank N, Lohr S, Reis A, et al. Evidence for genetic overlap between adult onset Still's disease and hereditary periodic fever syndromes. Rheumatol Int. 2018;38(1):111-20.

19. Karczewski KJ, Francioli LC, Tiao G, Cummings BB, Alföldi J, Wang Q, et al. Variation across 141,456 human exomes and genomes reveals the spectrum of loss-of-function intolerance across human protein-coding genes. BioRxiv. 2019; online, pre-accepted.

20. Witt M, Meier J, Hammitzsch A, Proft F, Schulze-Koops H, Grunke M. Disease burden, disease manifestations and current treatment regimen of the SAPHO syndrome in Germany: results from a nationwide patient survey. Semin Arthritis Rheum. 2014:43(6):745-50

21. Mossner R, Frambach Y, Wilsmann-Theis D, Lohr S, Jacobi A, Weyergraf A, et al. Palmoplantar Pustular psoriasis is associated with missense variants in CARD14, but not with loss-of-function mutations in IL36RN in European patients. J Invest Dermatol. 2015;135(10):2538-41.

22. Xiao G, Cheng H, Cao H, Chen K, Tu Y, Yu S, et al. Critical role of filaminbinding LIM protein 1 (FBLP-1)/migfilin in regulation of bone remodeling. J Biol Chem. 2012;287(25):21450-60.

\section{Publisher's Note}

Springer Nature remains neutral with regard to jurisdictional claims in published maps and institutional affiliations.

Ready to submit your research? Choose BMC and benefit from:

- fast, convenient online submission

- thorough peer review by experienced researchers in your field

- rapid publication on acceptance

- support for research data, including large and complex data types

- gold Open Access which fosters wider collaboration and increased citations

- maximum visibility for your research: over $100 \mathrm{M}$ website views per year

At BMC, research is always in progress.

Learn more biomedcentral.com/submissions 